\title{
Analytical method for determining quantum well exciton properties in a magnetic field
}

\author{
Piotr Stępnicki, ${ }^{1}$ Barbara Piętka, ${ }^{2}$ François Morier-Genoud, ${ }^{3}$ Benoît Deveaud, ${ }^{3}$ and Michał Matuszewski ${ }^{1}$ \\ ${ }^{1}$ Instytut Fizyki Polskiej Akademii Nauk, Aleja Lotników 32/46, 02-668 Warsaw, Poland \\ ${ }^{2}$ Instytut Fizyki Doświadczalnej, Wydziat Fizyki, Uniwersytet Warszawski, ul. Pasteura 5, Warsaw, Poland \\ ${ }^{3}$ Ecole Polytechnique Fédérale de Lausanne (EPFL), Lausanne, Switzerland
}

(Received 15 January 2015; revised manuscript received 14 April 2015; published 5 May 2015)

\begin{abstract}
We develop an analytical approximate method for determining the Bohr radii of Wannier-Mott excitons in thin quantum wells under the influence of magnetic field perpendicular to the quantum well plane. Our hybrid variational-perturbative method allows us to obtain simple closed formulas for exciton binding energies and optical transition rates. We confirm the reliability of our method through exciton-polariton experiments realized in a GaAs/AlAs microcavity with an $8 \mathrm{~nm} \operatorname{In}_{x} \mathrm{Ga}_{1-x}$ As quantum well and magnetic field strengths as high as $14 \mathrm{~T}$.
\end{abstract}

DOI: 10.1103/PhysRevB.91.195302

PACS number(s): 71.35.Ji, 71.36.+c, 73.21.Fg, 73.43.Cd

\section{INTRODUCTION}

Excitons are important from the point of view of both fundamental research and real life applications. The concept of an exciton as a localized electronic excitation is an essential part of modern physics of solids, and is widely used for explanation of optical properties of many materials. Recently the topic of strong coupling between photons and excitons, which leads to the creation of a new quasiparticle called the exciton-polariton [1], attracted much attention. Advances in achieving degenerate gases of excitons [2] and excitonpolaritons [3] opened up a new perspective for the field in connection with physics of ultracold quantum systems. Studies of excitons in magnetic field are important in the context of possible applications for spinoptronics [4-6].

The important task of calculating basic properties of excitons, including the binding energy and Bohr radius, can be tackled by numerous methods. While the most successful ones appear to make use of the variational method [7-17], schemes based on perturbation theory $[16,18,19]$, fractional dimensionality [15,20-22], or other sophisticated methods [14,19,23] were used, as well as direct numerical calculations. Depending on the desired accuracy, models may be based on the oneband effective mass approximation, or include the effects of valence-band mixing, subband mixing, band nonparabolicity and anisotropy, or difference in dielectric constants in the medium $[11,17,24,25]$.

In this paper, we propose an analytical method for calculating the exciton Bohr radius, energy, and optical transitions rates for excitons in quantum wells (QWs) in the presence of magnetic field perpendicular to the QW plane. We restrict our considerations to the simple one-band effective-mass Hamiltonian. Our hybrid variational-perturbative method allows us to obtain simple closed formulas for these quantities valid for a surprisingly wide range of magnetic field strengths. Typically, these formulas require only one external parameter, such as the exciton Bohr radius at zero magnetic field. To our knowledge, so far no analytical results have been obtained for the QW exciton problem beyond determining the quadratic diamagnetic shift. As we demonstrate below, our method is accurate far beyond this regime, up to magnetic fields of the order of $10 \mathrm{~T}$. We confirm the reliability of our method by comparing its predictions to the results of exciton-polariton experiments realized in a GaAs- $\operatorname{In}_{x} \mathrm{Ga}_{1-x}$ As microcavity with an $8 \mathrm{~nm}$ quantum well, where the exciton energy and oscillator strength are retrieved from the momentum dispersion of the upper and lower polariton branches.

\section{MODEL}

We consider Wannier-Mott excitons in a type-I semiconductor quantum well. The magnetic field is applied in the direction perpendicular to the quantum well. We assume tight confinement in a thin quantum well, with the energy spacing between subbands much larger than the energy of the electron and hole motion in the quantum well plane. This allows us to restrict our considerations to single electron and hole subbands only. Furthermore, we neglect the effect mixing of light and heavy holes [26] and apply the effective mass approximation to the electron and hole bands. With the above approximations the exciton can be treated as a hydrogen-like structure in a homogeneous medium. For simplicity, we restrict our considerations to the case of excitons with zero momentum in the QW plane. We note that the presence of a magnetic field can lead to a dramatic change of the exciton structure [27] in the case of high exciton momentum. The effective exciton Hamiltonian can be expressed as

$$
\hat{H}=\hat{H}_{e x}+\hat{H}_{h}+\hat{H}_{e},
$$

where

$$
\begin{aligned}
\hat{H}_{v}= & -\frac{\hbar^{2}}{2 m_{v}} \frac{\partial^{2}}{\partial z_{v}^{2}}+V_{v}-\mu_{B} g_{v} s_{v} B, \quad v=e, h, \\
\hat{H}_{e x}= & -\frac{\hbar^{2}}{2 \mu}\left[\frac{1}{\rho} \frac{\partial}{\partial \rho}\left(\rho \frac{\partial}{\partial \rho}\right)-\frac{\rho^{2}}{4 L^{4}}\right]+\frac{L_{z}^{2}}{2 \mu} \\
& +\frac{\hbar}{2 \eta L^{2}} L_{z}-\frac{e^{2}}{4 \pi \epsilon \epsilon_{0} \sqrt{\rho^{2}+\left(z_{e}-z_{h}\right)^{2}}},
\end{aligned}
$$

where $\rho$ is the distance between electron and hole in the QW plane, $V_{e(h)}$ is the electron (hole) confinement potential, $\mu^{-1}=m_{e}^{-1}+m_{h}^{-1}, \eta^{-1}=m_{e}^{-1}-m_{h}^{-1}, L=\sqrt{\frac{\hbar}{e B}}$, and $L_{z}=$ $-i \hbar(\partial / \partial \theta)$ is the operator of orbital angular momentum along the axis $z$ perpendicular to the QW plane. For simplicity we assume that the confinement potentials are of the form

$$
V_{v}\left(z_{v}\right)= \begin{cases}0 & \text { for }-\frac{d}{2}<z_{v}<\frac{d}{2}, \\ V_{v}^{0} & \text { otherwise }\end{cases}
$$


where $V_{v}^{0}$ and $d$ are the QW depth and width, respectively.

Since the Hamiltonian (2) cannot be solved analytically even in the absence of the magnetic field, a large number of approximate methods have been developed in the past to determine the energy levels and other properties of excitons. Among these, the variational approach appears to be most successful in terms of accuracy and simplicity, although its reliability depends on the somewhat arbitrary choice of the variational ansatz.

Moreover, in most practical cases even the approximate variational problem has to be tackled by numerical methods. In the following we show that if the Bohr radius or the energy of the exciton in the absence of magnetic field is known (e.g., from other numerical calculations or experimental measurements), the exciton properties in the presence of magnetic field can be determined by much simpler means. This includes, in the lowest level of approximation, a simple analytical formula that gives accurate results in a very wide range of magnetic field strengths.

\section{METHOD}

\section{A. Variational approximation}

In the case of tight confinement the wave function of the electron-hole system can be separated in directions perpendicular and parallel to the quantum well plane. In the case of a $1 s$ exciton we apply a variational ansatz in the form [7]

$$
\psi\left(\rho, z_{e}, z_{h}\right)=\sqrt{\frac{2}{\pi}} \frac{1}{a} e^{-\rho / a} U_{e}\left(z_{e}\right) U_{h}\left(z_{h}\right),
$$

where $a$ is the exciton Bohr radius and $U_{e}, U_{h}$ are the subband electron and hole wave functions. We note that the method can be easily generalized to the case of higher hydrogen-like exciton states. Evaluating the expectation value of the above Hamiltonian (2) with the ansatz (4) and differentiating with respect to $a$ gives an equation that corresponds to the energy minimum

$$
1-\frac{3}{8}\left(\frac{a}{L}\right)^{4}=\frac{8 \mu}{\hbar^{2}} \int_{0}^{\infty} \rho\left(1-\frac{\rho}{a}\right) e^{-2 \rho / a} V(\rho) d \rho,
$$

where $V(\rho)$ is given by

$$
V(\rho)=\frac{e^{2}}{4 \pi \epsilon \epsilon_{0}} \iint d z_{e} d z_{h} \frac{U_{e}^{2} U_{h}^{2}}{\sqrt{\rho^{2}+\left(z_{e}-z_{h}\right)^{2}}} .
$$

Therefore finding the approximate ground state of the Hamiltonian is equivalent to solving Eq. (5).

Up to now we have followed the standard variational procedure. Solving Eq. (5) requires fixing the perpendicular wave functions $U_{e}, U_{h}$, determining the effective Coulomb potential $V(\rho)$, and solving the integral equation given by (5) by numerical means.

\section{B. Expansion}

The general idea of simplifying the problem of solving Eq. (5) is to approximate its right-hand side by a polynomial of a degree smaller than 4 (so that we obtain an exactly solvable polynomial equation) in the variable $a$. The expansion of the right-hand side can be done in many different ways, and here we present the one that appears to provide the greatest accuracy.

The idea is realized in several steps. First, we change the integral variable to $x=\rho / a$ on the right-hand side of Eq. (5) which allows us to eliminate $a$ from the integral everywhere except $V(a x)$. Let $a_{0}$ be the solution to the Eq. (5) at zero magnetic field, $a(0)=a_{0}$. We now write the effective potential as $V(\rho)=V(a x)=V(a, x)$ and expand it in the variable $a$ at the point $a=a_{0}$. We reformulate Eq. (5) to obtain

$$
\begin{aligned}
\frac{\hbar^{2}}{8 \mu}\left[1-\frac{3}{8}\left(\frac{a}{L}\right)^{4}\right] & a^{2} \underbrace{\int_{0}^{\infty} x(1-x) e^{-2 x} V\left(a_{0}, x\right) d x}_{D_{0}} \\
& +a^{2}\left(a-a_{0}\right) \underbrace{\left.\int_{0}^{\infty} x(1-x) e^{-2 x} \frac{\partial V(a, x)}{\partial a}\right|_{\left(a_{0}, x\right)} d x}_{D_{1}} \\
& +\cdots .
\end{aligned}
$$

The above expansion requires several comments to explain its physical meaning. The main approximation of our method consists in neglecting most of the terms in the series, retaining only the first one or two. It is important to note that this approximation is not simply equivalent to neglecting higher order effects of the magnetic field, since the right-hand side contains only terms that are related to the Coulomb potential. This will become more apparent later, when we show that the accuracy of the method extends far beyond determining the quadratic diamagnetic shift. On the other hand, the approximation is not equivalent to treating the Coulomb potential as a perturbation. In fact, the effect of $V(\rho)$ is fully accounted for in the limit of vanishing magnetic field when $a=a_{0}$. The idea behind the above expansion is that by introducing the variable $x$ that scales together with the exciton radius we take into account that the wave function shrinks in the presence of magnetic field. This leads to the change of the effective Coulomb potential in the rescaled variable $x$. It is this change of the effective potential that is taken into account perturbatively in Eq. (7).

The $D_{0}, D_{1}$ integrals can be written as

$$
\begin{aligned}
D_{0}= & \frac{e^{2}}{4 \pi \epsilon \epsilon_{0}} \int_{0}^{\infty} d x \int_{\mathbb{R}^{2}} d z_{e} d z_{h} x(1-x) e^{-2 x} \\
& \times \frac{U_{e}^{2} U_{h}^{2}}{\sqrt{\left(x a_{0}\right)^{2}+\left(z_{e}-z_{h}\right)^{2}}}, \\
D_{1}= & -\frac{e^{2}}{4 \pi \epsilon \epsilon_{0}} \int_{0}^{\infty} d x \int_{\mathbb{R}^{2}} d z_{e} d z_{h} a_{0} x^{3}(1-x) e^{-2 x} \\
& \times \frac{U_{e}^{2} U_{h}^{2}}{\left[\left(x a_{0}\right)^{2}+\left(z_{e}-z_{h}\right)^{2}\right]^{\frac{3}{2}}} d x d z_{e} d z_{h} .
\end{aligned}
$$

After computing the $D_{0}$ and $D_{1}$ integrals for given functions $U_{e}, U_{h}$ one obtains a fourth-order polynomial in $a$ which describes the behavior of $a(B)$. Taking into account that $a_{0}$ satisfies Eq. (5) for $L=\infty$ the value for $D_{0}$ can be obtained 


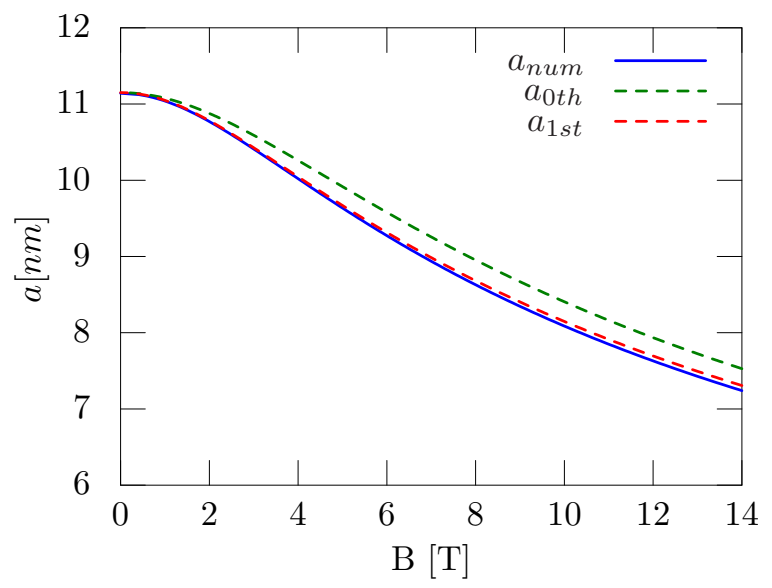

FIG. 1. (Color online) Bohr radius $a$ of the exciton versus magnetic field strength for an InGaAs quantum well. $a_{\text {num }}$ corresponds to exact numerical solution of Eq. (5), $a_{1 s t}$ to the approximation including both $D_{0}$ and $D_{1}$ terms in Eq. (7), and $a_{0 t h}$ is the zeroth-order approximation including the $D_{0}$ term only, Eq. (11). The subband functions $U_{e}, U_{h}$ were chosen to be the solutions of the 1D finite potential barrier problem.

from

$$
D_{0}=\lim _{L \rightarrow \infty} \frac{\hbar^{2}}{8 \mu a_{0}^{2}}\left[1-\frac{3}{8}\left(\frac{a_{0}}{L}\right)^{4}\right]=\frac{\hbar^{2}}{8 \mu a_{0}^{2}} .
$$

Numerical checks for a realistic GaAs quantum well exciton indicate that the linear approximation, including both $D_{0}$ and $D_{1}$ terms, gave almost perfect results for a realistic example of a thin quantum well with a tight exciton confinement; see Fig. 1. In this calculation, we used cosine functions for the lowest subband electron and hole wave functions $U_{e}\left(z_{e}\right)$ and $U_{h}\left(z_{h}\right)$. However, any form of subband wave functions can be easily incorporated in the calculation. We stress that computing the coefficients $D_{0}$ and $D_{1}$ is much simpler than solving the integral variational equation (5).

Moreover, we found that the approximation including the $D_{0}$ term only gives also very accurate results for Bohr radius $a$. In this case, a simple analytical formula can be given for $a(B)$ :

$$
a(B)=a_{0} \sqrt{2}\left[\sqrt{1+\frac{3}{2}\left(\frac{e^{2} a_{0}^{4} B^{2}}{\hbar^{2}}\right)}+1\right]^{-\frac{1}{2}} .
$$

The formula (11) applies to any material and confinement potential, but the confinement must be tight enough for the ansatz (4) to be correct. The only needed parameter is the exciton radius at zero magnetic field, $a_{0}$.

We performed calculations for $B$ as high as $15 \mathrm{~T}$ to compare formula (11) and the exact numerical solution of (5). For all considered cases the quantitative agreement between these solutions was very good.

\section{Exciton energies and optical transition rates}

The approximate formula for the exciton Bohr radius $a$ dependence on the magnetic field allows for calculation of the exciton energy and optical transition rates. One may easily find an analytical formula for exciton energy using Eq. (5) and expansion of $V(a x)$. Using the formula (11) for $a$ one obtains

$$
\begin{aligned}
E_{X}= & V_{0}+\sqrt{2} V_{1} a_{0}\left[\sqrt{1+\frac{3}{2}\left(\frac{e^{2} a_{0}^{4} B^{2}}{\hbar^{2}}\right)}+1\right]^{-\frac{1}{2}} \\
& -\frac{\hbar^{2}}{2 \mu a_{0}^{2}} \sqrt{1+\frac{3}{2}\left(\frac{e^{2} a_{0}^{4} B^{2}}{\hbar^{2}}\right)}-\frac{\hbar e B}{\mu}-g \mu_{B} s B
\end{aligned}
$$

where

$$
\begin{aligned}
& V_{0}=4 \int_{0}^{\infty} e^{-2 x}\left[V\left(a_{0} x\right)+\left.a_{0} \frac{\partial V(a, x)}{\partial a}\right|_{a_{0}}\right] x d x, \\
& V_{1}=\left.4 \int_{0}^{\infty} e^{-2 x} \frac{\partial V(a, x)}{\partial a}\right|_{a_{0}} x d x .
\end{aligned}
$$

In the above formulas we included the first-order derivatives of $V$, which give a much more important contribution than in the Bohr radius equation (7). At the same time, inclusion of these terms in above energy formula does not lead to significant complication of the calculation.

The change of the exciton size influences also the exciton transition dipole moment and consequently the optical exciton creation rates. In the case of an experiment performed in a microcavity, this rate is proportional to the splitting of lower and upper polariton lines $[1,28]$. In the case of Wannier-Mott excitons the exciton transition rate is, with a high degree of accuracy, proportional to the norm of the exciton wave function for zero relative electron-hole coordinate [29], $|\psi(\rho=0)|$. The approximate expression for the increase of exciton Rabi splitting in magnetic field is then

$$
\begin{aligned}
\Omega(B) & =\Omega_{0} \frac{a_{0}}{a(B)}=\Omega_{0} \frac{\sqrt{3} e a_{0}^{2} B}{2 \hbar\left[\sqrt{1+\frac{3}{2}\left(\frac{e^{2} a_{0}^{4} B^{2}}{\hbar^{2}}\right)}-1\right]^{\frac{1}{2}}} \\
& =\frac{\Omega_{0}}{\sqrt{2}}\left[\sqrt{1+\frac{3}{2}\left(\frac{e^{2} a_{0}^{4} B^{2}}{\hbar^{2}}\right)}+1\right]^{\frac{1}{2}},
\end{aligned}
$$

where $\Omega_{0}$ and $a_{0}$ are the Rabi splitting and exciton radius at zero magnetic field.

\section{NUMERICAL AND EXPERIMENTAL TESTS}

The accuracy and reliability of the method was tested through direct comparison with both the numerical solutions of the full variational problem (5) and with experimental measurements of the energy and Rabi splitting of excitonpolaritons in a semiconductor microcavity containing an $8 \mathrm{~nm}$ single $\mathrm{In}_{0.04} \mathrm{Ga}_{0.96} \mathrm{As}$ quantum well $(\mathrm{QW})$. The $\mathrm{QW}$ was placed in the GaAs $\lambda$ microcavity between two GaAs/AlAs distributed Bragg reflectors. The vacuum-field Rabi splitting $\Omega$ at zero magnetic field was approximately $3.5 \mathrm{meV}$. The sample was placed in a helium bath cryostat and in the magnet bore providing magnetic field up to $14 \mathrm{~T}$ perpendicular to the sample plane. The photoluminescence (PL) was excited by a continuous wave laser with the energy above the band gap of the material $(1.72 \mathrm{eV})$. The PL signal was propagating free in space to our detection setup which allowed us to collect 


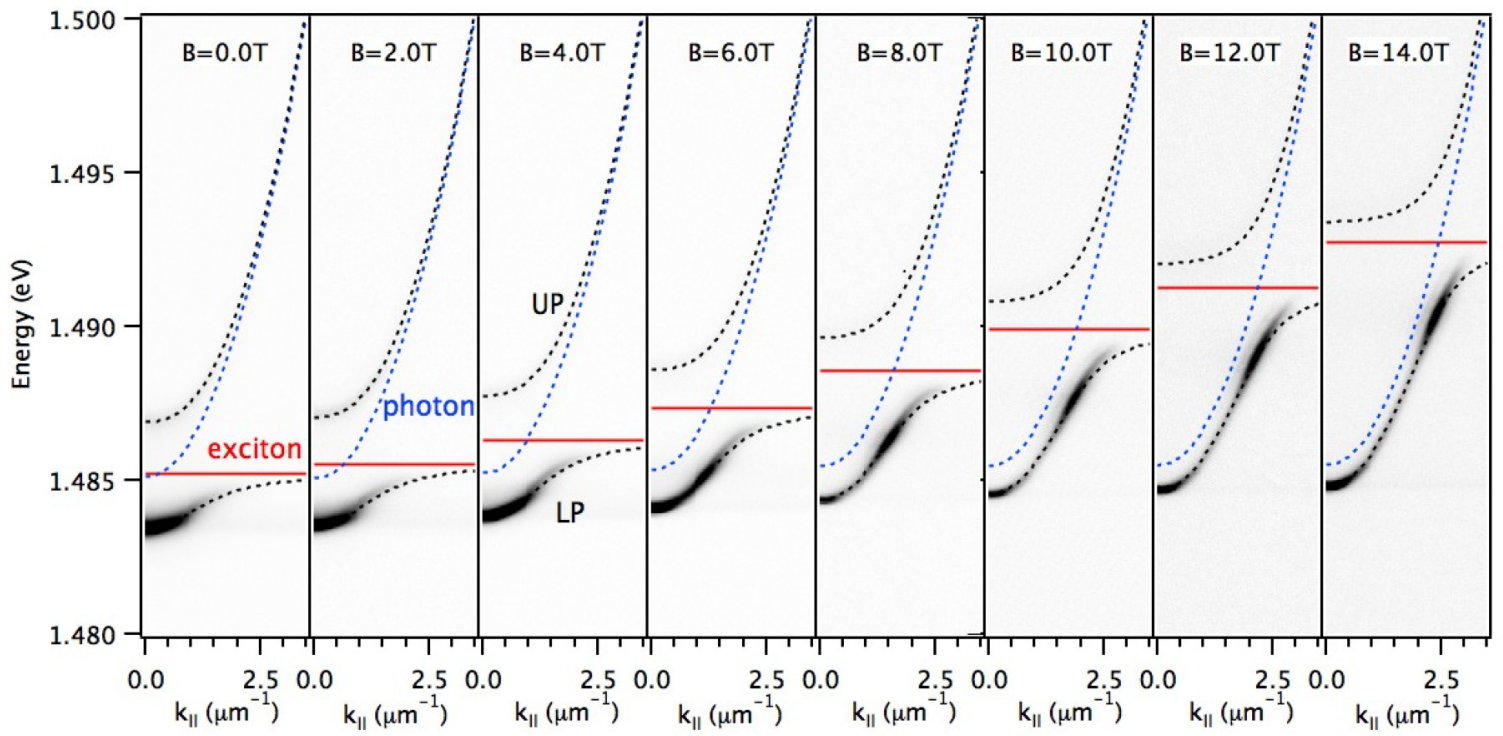

FIG. 2. (Color online) Photoluminescence spectra of exciton-polaritons at magnetic fields 0, 2, 4, 6, 8, 10, 12, and 14 T. The lower (LP) and upper (UP) polariton branches are marked (dashed lines) together with fitted bare excitonic (red solid line) and photonic (blue dashed) resonances. The color PL intensity scale is from white (low intensity) to black (high intensity). The respective images are not in the same intensity scale. The total intensity is decreasing in magnetic field [31].

the exciton-polariton dispersion (energy vs in-plane polariton momentum). The details of the sample structure can be found in Ref. [30] and the experimental setup together with detailed description of the performed experiment in Ref. [31]. We detect the PL in a continuous sweep of magnetic field from 0 to 14 T. Figure 2 illustrates photoluminescence spectra for a few values of magnetic field strength. We observe lower and upper polariton branches. Fitting the simple model of two coupled oscillators [1] to the experimental data we can obtain the energy of the excitonic and photonic bare resonances (marked directly in Fig. 2) and the value of the Rabi splitting $\Omega$. This allows to obtain unprecedented precision on the variations of the exciton energy and oscillator strength.

In Fig. 1 we show the exciton Bohr radius as predicted by the exact numerical solution of Eq. (5) and by our analytical approximation in the cases when only one or two terms were kept in the expansion (7). The parameters of the quantum well correspond to the sample used in the experiment described above. The only external parameter in the calculations was the value of exciton radius at zero magnetic field, $a_{0}$. The agreement between the numerical results and the approximation with both $D_{0}$ and $D_{1}$ terms included is almost perfect. When only the $D_{0}$ term is included, the analytical formula (11) provides also a very accurate prediction, with no more than a few percent discrepancy up to magnetic field strength of $15 \mathrm{~T}$. It is important to note that the accuracy is significantly better than in the case of standard perturbation method including only quadratic terms in the magnetic field strength (or quadratic diamagnetic shift), which deviate from the correct dependence already for fields higher than $4 \mathrm{~T}$. Our approximation works well for a broad range of magnetic fields because its accuracy depends on the variation of the value $a(B)$ rather that on the variation of $B$.

In Figs. 3 and 4 we compare the predictions of our model to the energy and exciton radius obtained from experimental measurements described above. The energy of the $1 s$ heavy hole (1sHH) exciton ground state, shown in Fig. 3, is recovered with a very high accuracy up to the regime of relatively strong magnetic field $(14 \mathrm{~T})$. Here again we used only the single external parameter $a_{0}$. It is evident that the accuracy of the simple analytical formula (12) extends far beyond predicting the strength of the quadratic diamagnetic shift. The spin term is neglected as its influence is very small. The dependence of the polariton Rabi splitting is shown in Fig. 4, with another parameter $\Omega_{0}$. The accuracy of the analytical prediction is also very good up to the fields of around $10 \mathrm{~T}$. The discrepancy at $B \approx 1-2 \mathrm{~T}$ is due to the influence of the $2 \mathrm{sHH}$ exciton resonance which disturbs the shape of the polariton branches. At magnetic fields stronger than $10 \mathrm{~T}$ the accuracy is gradually lost, which can be also observed in the slight deviation of the

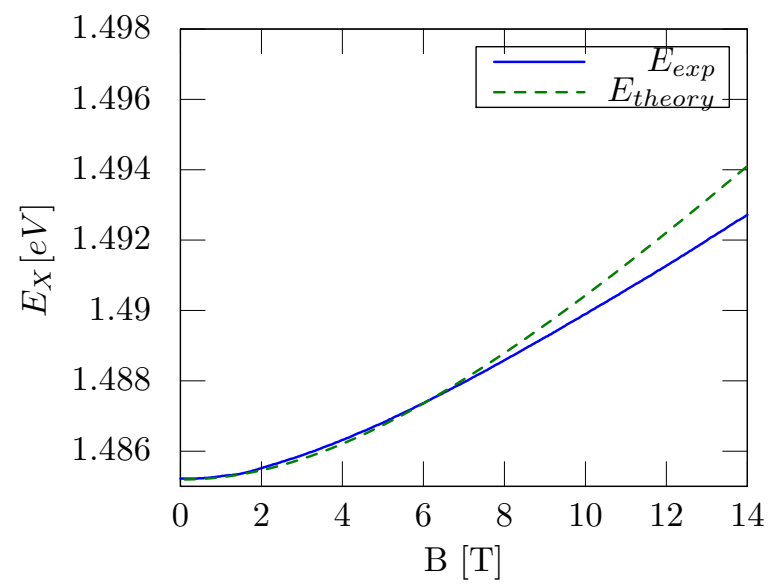

FIG. 3. (Color online) Comparison of the exciton energy for InGaAs quantum well from the experiment (solid line) and the predictions of the analytical formula (12) (dashed line). 


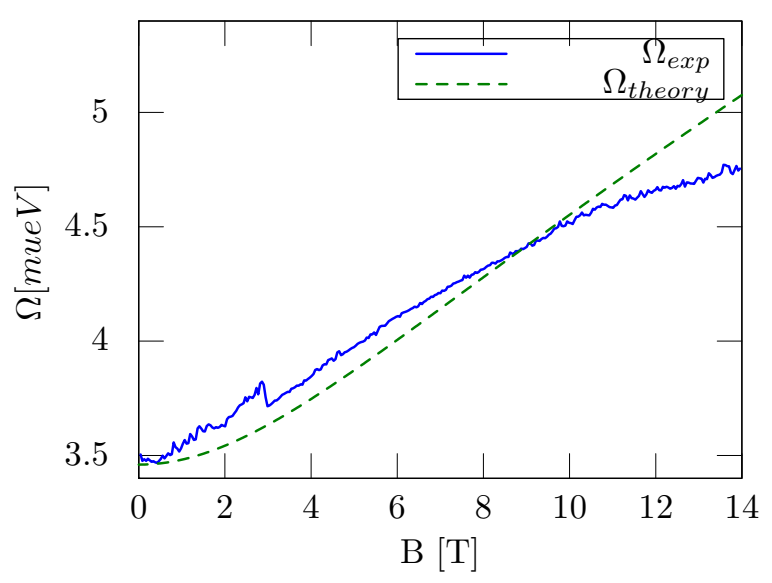

FIG. 4. (Color online) Comparison of the exciton-polariton Rabi splitting for InGaAs quantum well from the experiment (solid line) and the predictions of the analytical formula (14) (dashed line).

exciton energy in Fig. 3. Nevertheless, the ability to predict the exciton energies and transition rates extends to surprisingly high values of the magnetic field strength.

\section{CONCLUSIONS}

In conclusion, we developed an analytical approximate method for determining the Bohr radii of Wannier-Mott excitons in thin quantum wells under the influence of magnetic field perpendicular to the quantum well plane. Our hybrid variational-perturbative method allows us to obtain simple closed formulas for exciton binding energies and optical transition rates. We confirm the reliability of our method in exciton-polariton experiments realized in a GaAs/AlAs microcavity with an $8 \mathrm{~nm} \operatorname{In}_{x} \mathrm{Ga}_{1-x}$ As quantum well and magnetic field strengths as high as $14 \mathrm{~T}$.

\section{ACKNOWLEDGMENTS}

P.S. and M.M. acknowledge support from National Science Center Grant No. DEC-2011/01/D/ST3/00482. B.P. acknowledges support from National Science Center Grant No. 2011/01/D/ST7/04088. The experimental work was possible thanks to the Physics Facing Challenges of the XXI Century grant at Warsaw University, Poland. We would like to thank M. R. Molas and A. A. L. Nicolet for help in the experimental work and M. Potemski for the possibility of performing experiments in magnetic field up to $14 \mathrm{~T}$, and for fruitful discussions.
[1] J. J. Hopfield, Phys. Rev. 112, 1555 (1958); C. Weisbuch, M. Nishioka, A. Ishikawa, and Y. Arakawa, Phys. Rev. Lett. 69, 3314 (1992); A. V. Kavokin, J. J. Baumberg, G. Malpuech, and F. P. Laussy, Microcavities (Oxford University Press, Oxford, 2007).

[2] D. W. Snoke, J. P. Wolfe, and A. Mysyrowicz, Phys. Rev. B 41, 11171 (1990); A. A. High, J. R. Leonard, A. T. Hammack, M. M. Fogler, L. V. Butov, A. V. Kavokin, K. L. Campman, and A. C. Gossard, Nature (London) 483, 584 (2012); M. Alloing, M. Beian, D. Fuster, Y. Gonzalez, L. Gonzalez, R. Combescot, M. Combescot, and F. Dubin, Europhys. Lett. 107, 10012 (2014).

[3] J. Kasprzak, M. Richard, S. Kundermann, A. Baas, P. Jeambrun, J. M. J. Keeling, F. M. Marchetti, M. H. Szymańska, R. André, J. L. Staehli, V. Savona, P. B. Littlewood, B. Deveaud, and L. S. Dang, Nature (London) 443, 409 (2006); S. Christopoulos, G. Baldassarri Hoger von Högersthal, A. J. D. Grundy, P. G. Lagoudakis, A. V. Kavokin, J. J. Baumberg, G. Christmann, R. Butté, E. Feltin, J.-F. Carlin, and N. Grandjean, Phys. Rev. Lett. 98, 126405 (2007).

[4] G. Grosso, J. Graves, A. T. Hammack, A. A. High, L. V. Butov, M. Hanson, and A. C. Gossard, Nat. Photonics 3, 577 (2009).

[5] I. A. Shelykh, G. Pavlovic, D. D. Solnyshkov, and G. Malpuech, Phys. Rev. Lett. 102, 046407 (2009).

[6] D. Bajoni, E. Semenova, A. Lemaître, S. Bouchoule, E. Wertz, P. Senellart, S. Barbay, R. Kuszelewicz, and J. Bloch, Phys. Rev. Lett. 101, 266402 (2008).

[7] G. Bastard, E. E. Mendez, L.L. Chang, and L. Esaki, Phys. Rev. B 26, 1974 (1982).

[8] R. L. Greene and K. K. Bajaj, Phys. Rev. B 31, 6498 (1985).
[9] J. Cen, S. V. Branis, and K. K. Bajaj, Phys. Rev. B 44, 12848 (1991).

[10] R. L. Greene, K. K. Bajaj, and D. E. Phelps, Phys. Rev. B 29, 1807 (1984).

[11] Z. Barticevic, M. Pacheco, C. A. Duque, and L. E. Oliveira, J. Phys.: Condens. Matter 14, 1021 (2002).

[12] G. Bastard, Wave Mechanics Applied to Semiconductor Heterostructures (Les Editions de Physique, Paris, 1988).

[13] J. Kossut, J. K. Furdyna, and M. Dobrowolska, Phys. Rev. B 56, 9775 (1997).

[14] T. M. Rusin, J. Phys.: Condens. Matter 12, 575 (2000).

[15] E. Reyes-Gmez, L. E. Oliveira, and M. de Dios-Leyva, Semicond. Sci. Technol. 19, 699 (2004).

[16] Kyu-Seok Lee, Y. Aoyagi, and T. Sugano, Phys. Rev. B 46, 10269 (1992).

[17] D. B. Tran Thoai, R. Zimmermann, M. Grundmann, and D. Bimberg, Phys. Rev. B 42, 5906 (1990).

[18] S. N. Walck and T. L. Reinecke, Phys. Rev. B 57, 9088 (1998).

[19] X. L. Zheng, D. Heiman, and B. Lax, Phys. Rev. B 40, 10523 (1989).

[20] H. Mathieu, P. Lefebvre, and P. Christol, Phys. Rev. B 46, 4092 (1992).

[21] A. Thilagam, Physica B 262, 390 (1999).

[22] R. A. Escorcia, J. Sierra-Ortega, I. D. Mikhailov, and F. J. Betancur, Physica B 355, 255 (2005).

[23] R. P. Leavitt and J. W. Little, Phys. Rev. B 42, 11774 (1990).

[24] L. C. Andreani and A. Pasquarello, Phys. Rev. B 42, 8928 (1990).

[25] U. Ekenberg and M. Altarelli, Phys. Rev. B 35, 7585 (1987).

[26] G. E. W. Bauer and T. Ando, Phys. Rev. B 38, 6015 (1988). 
[27] Y. E. Lozovik, I. V. Ovchinnikov, S. Y. Volkov, L. V. Butov, and D. S. Chemla, Phys. Rev. B 65, 235304 (2002).

[28] J. D. Berger, O. Lyngnes, H. M. Gibbs, G. Khitrova, T. R. Nelson, E. K. Lindmark, A. V. Kavokin, M. A. Kaliteevski, and V. V. Zapasskii, Phys. Rev. B 54, 1975 (1996).

[29] R. J. Elliot, Phys. Rev. 108, 1384 (1957); M. Sugawara, N. Okazaki, T. Fujii, and S. Yamazaki, Phys. Rev. B 48, 8848 (1993).
[30] O. El Daif, A. Baas, T. Guillet, J.-P. Brantut, R. Idrissi Kaitouni, J. L. Staehli, F. Morier-Genoud, and B. Deveaud, Appl. Phys. Lett. 88, 061105 (2006).

[31] B. Piętka, D. Zygmunt, M. Król, M. R. Molas, A. A. L. Nicolet, F. Morier-Genoud, J. Szczytko, J. Łusakowski, P. Zięba, I. Tralle, P. Stępnicki, M. Matuszewski, M. Potemski, and B. Deveaud, Phys. Rev B 91, 075309 (2015). 